\title{
Heart-rate variability during gravitational transition in doxorubicin-induced cardiomyopathic guinea pig
}

\author{
Pattara Rattanawong ${ }^{\mathrm{a}}$, Wasawat Vutthikraivit ${ }^{\mathrm{a}}$, Attawit Charoensri ${ }^{\mathrm{a}}$, Tachapong Ngarmukos ${ }^{\mathrm{a}}$, \\ Anusak Kijtawornrat ${ }^{\mathrm{b}}$, Ichiro Miyoshi ${ }^{\mathrm{c}}$, Jonggonnee Wattanapermpool ${ }^{\mathrm{d}}$, Tepmanas Bupha-Intr ${ }^{\mathrm{d}, *}$ \\ ${ }^{a}$ Faculty of Medicine Ramathibodi Hospital, Bangkok 10400 Thailand \\ b Faculty of Veterinary Science, Chulalongkorn University, Bangkok 10330 Thailand \\ c Department of Comparative and Experimental Medicine and Centre for Experimental Animal Science, \\ Nagoya City University Graduate School of Medical Sciences, Nagoya, Japan \\ d Department of Physiology, Faculty of Science Mahidol University, Bangkok 10400 Thailand
}

*Corresponding author, e-mail: tepmanas.bup@mahidol.ac.th

Received 9 Jan 2015

Accepted 13 Oct 2015

\begin{abstract}
Changes in gravitational force cause no serious problems in healthy persons but might induce vital disturbances in a person with cardiovascular abnormalities. To evaluate the potential of neuro-cardiogenic disruption in a heart at risk during abrupt gravitational transition, heart rate variability of doxorubicin-induced cardiomyopathic (CM) guinea pigs were monitored during a parabolic flight. An electrocardiogram was continuously recorded during the change from normal gravity to $1.8 \mathrm{~g}$ (hypergravity) and then to $0 \mathrm{~g}$ (microgravity). Time domain heart rate variability indices, including standard deviation of intervals (RRSD) and the square root of mean squared differences of successive intervals (rMSSD), corrected QT interval (QTc), and short-term QT variability (STVQT) were compared. At normal gravity, decreases in RRSD and rMSSD with prolonged QTc interval were observed in the CM group compared to controls. Further significant reduction of RRSD was seen only in CM group during hypergravity, while a decrease in rMSSD was only found in the control group, indicating an increase in arrhythmic risk. A significant increase in STVQT was present only in the control group in microgravity, suggesting a possibility of decreased sympathetic activation to the heart. Interestingly, prolonged QTc interval in the CM group under normal gravity was reversed compared to that of the control group under microgravity. These findings indicate that a gravitational force change from normal to hypergravity acutely increases arrhythmia risk in doxorubicin-induced CM guinea pigs, which is reduced under microgravity. These preliminary findings in animal model are pertinent if commercial space travel aims to become commonplace and safe.
\end{abstract}

KEYWORDS: microgravity, hypergravity, parabolic flight

\section{INTRODUCTION}

Space travel will become practical in the near future. Knowledge regarding physiological responses during gravitational transition is, however, limited only to healthy people. Stress to either haemodynamic or neurohormonal changes during space journey possibly elicit the non-symptomatic defect and aggravate the disease severity leading to detrimental results. In a healthy person, the mechanism underlying acute changes during microgravity exposure are likely to be similar to those observed during change in the body position from upright to supine. The supine pre-launch position with the lower limbs raised above thoraco-abdominal coronal plane initiates a fluid shift from the lower limbs to the torso and head ${ }^{1}$. As a result, an increased venous return causes immediate increase in stroke volume, and cardiac output. In response, an increase in heart rate variability seen during acute simulated microgravity indicates a potential increase in parasympathetic activation ${ }^{2}$. Conversely, a transition to hypergravity-induced reduction of left ventricular volume in standing upright position ${ }^{3}$ is associated with a decreased vagal stimulation ${ }^{4}$. However, the responses due to haemodynamic changes might be uncommon in the heart disease patients who already have high central venous pressure.

Beside haemodynamic disturbance, Petersen et al demonstrated an increase in cardiac output when entering microgravity even in the supine position ${ }^{5}$. Using lower body negative pressure induction, those changes in heart rate variability were still observed at microgravity ${ }^{4}$. Such neurohormonal changes constitute the major factor regulating cardiovascular response during a gravitational transi- 
tion. By monitoring the rat in recumbent position, an increase in blood pressure, but decrease in heart rate during exposure to microgravity ${ }^{6}$ supports the significance of cardio-neuronal effect during gravitational transition.

In the failing heart, a decrease in contractility results in decreases in stroke volume, cardiac output, and arterial blood pressure followed by an increase in sympathetic activity and release of catecholamines. Decreased parasympathetic activation in heart disease could be demonstrated from a reduction in heart rate variability ${ }^{7}$. The absence of a cardiovascular reflex moving from supine to standing in the patients with congestive heart failure ${ }^{8}$ however indicate an impairment of cardio-neuronal function. Suppression in heart rate response to blood volume expansion was also observed in a heart failure patient ${ }^{9}$. Desai et al demonstrated that an increase in heart rate variability during postural change from standing to supine, generally observed in healthy subjects, was absent in congestive heart failure patients ${ }^{10}$. Based on this information, the cardio-neuronal cooperation during gravitational transition might also be altered in a heart failure patient.

The aim of present study is then to preliminarily evaluate whether neuro-cardiogenic response during abruptly gravitational transition would be altered in animal heart disease model. Electrocardiographic parameters were compared between the heart of normal control and cardiomyopathic (CM) guinea pigs, induced by doxorubicin injection ${ }^{11}$, during gravitational transition in a parabolic flight. With the use of guinea pig model, the effect of positioning was minimized during the gravitational change. Electrocardiogram was continuously monitored during the three phases of parabolic flight, namely, normal, hyper-, and microgravity (kindly provided by Japan Aerospace Exploration Agency, JAXA). In brief, the results indicate that neurocardiogenic response was increased in CM guinea pigs during transition to hypergravity, while the response to microgravity was less.

\section{MATERIALS AND METHODS}

\section{Ethics}

All processes were performed in the centre of Experimental Animal Science, Department of Comparative and Experimental Medicine, Nagoya City University, Japan. The study was approved by the IACUC committee of Japan Aero Space and Exploration Agency, IACUC committee of Nagoya City University, and
IACUC committee of Faculty of science, Mahidol University.

\section{Animals}

Male Dunkin Hartley 12-week old guinea pigs, 500$550 \mathrm{~g}$, were divided into control and heart failure groups (4 animals per group). Cardiomyopathy was induced by doxorubicin injection after caffeine prefeeding. The cardiomyopathy group received oral feeding of caffeine solution $(15 \mathrm{mg} / \mathrm{ml})$ for two weeks with a dose of $120 \mathrm{mg} / \mathrm{kg}$ body weight on each of the first five days and subsequently with $90 \mathrm{mg} / \mathrm{kg}$ body weight on each of the last nine days. On day 12 of caffeine-feeding period, guinea pigs were intraperitoneally injected with a single dose of doxorubicin $(8 \mathrm{mg} / \mathrm{kg} \text { body weight })^{11}$. In the control group, normal saline was intraperitoneally injected on day 12 of experimentation but without caffeine pre-feeding. Animals were housed in a temperature-controlled room $\left(25^{\circ} \mathrm{C}\right)$ with access to food and water ad libitum. Cardiomyopathy was confirmed by histochemical examination of cardiac tissue following the parabolic flight.

\section{Experimental methods and data acquisition}

On day 15 , all guinea pigs were transported to the airport in a temperature-controlled vehicle $\left(25^{\circ} \mathrm{C}\right)$ and acclimatized for $2 \mathrm{~h}$ before the flight. Animals were anaesthetized with Zoletil:Xylazine (Tiletamine:Zolazepam) 5:60 mg/kg body weight twice, $1 \mathrm{~h}$ and a quarter of an hour before flight departure. Animals were placed on a bed at ventral recumbence and strapped to prevent free floating and movement during the transitional change of gravity. An electrode patch was attached for a bipolar transthoracic ECG between the points of $\mathrm{rV}_{2}$ and $\mathrm{V}_{2}$. During the parabolic flight, bipolar transthoracic ECG was continuously recorded using Biopac MP150 Data Acquisition Unit (Biopac Systems, Inc., Santa Barbara, CA) at $1000 \mathrm{~Hz}$ per channel and stored offline in a laptop computer. All animals were monitored and observed by video camera to ensure anaesthetic condition throughout the flight.

\section{Parabolic flight}

This study was conducted under the parabolic flight campaign 2010 by the Japan Aerospace Exploration Agency (JAXA). Flight was organized at Nagoya airport by the Japan Aerospace Exploration Agency and Diamond Air Service Inc., Nagoya. GulfstreamII (Turbo Jet) aircraft with an interior designed and especially fitted to accommodate the experiments. The parabolic flight incorporated 11-12 parabolic 
cycles with a 10-min interval between each cycle. Cruising altitude before the parabolic manoeuvre was $4570 \mathrm{~m}$ and the vertex of the parabola was $10670 \mathrm{~m}$. Following the free-fall trajectory, a short horizontal flight phase was carried out before performing successive parabolic manoeuvres. The signal from $g_{z}$ accelerometer was used to continuously measure gravity within the aircraft, perpendicular to the floor of the plane, during the three consecutive flight phases, namely, phase 1 , normal gravity lasting for $20 \mathrm{~s}$; phase 2, hypergravity $(1.8 \mathrm{~g})$ on ascending leg of the parabola lasting for $20 \mathrm{~s}$; and phase 3 , microgravity $(0 g)$ at the vertex of the parabola lasting for $20 \mathrm{~s}$. Unfortunately, the gravitation signal was not displayed during recovery period.

\section{Histopathological examination}

After the flight experiment, guinea pigs were sacrificed by $\mathrm{CO}_{2}$ inhalation. Heart specimens were fixed in $10 \%$ formalin phosphate buffer and embedded in paraffin wax. Sections of five micrometre thickness were stained with haematoxylin and eosin. Under a light microscope (400× magnification) histopathological examination was conducted to identify cardiomyocytes exhibiting cytoplasmic vacuolation, myofibrillar degeneration, and inflammatory cell infiltration. Severity of cardiac pathology was evaluated from 10 randomly selected fields and graded using a semi-quantitative method into grade 0 (no damage); grade 1 (less than $5 \%$ of cardiomyocytes); grade 2 (6-25\% of cardiomyocytes); grade 3 (26-49\% of cardiomyocytes); and grade 4 (more than $50 \%$ of cardiomyocytes). All examinations were performed independently by doubleblinded processing.

\section{DATA ACQUISITION AND STATISTICAL ANALYSIS}

\section{RR interval and heart rate variability}

ECGs were reviewed by visual inspection to exclude artefacts and ectopic beats. RR interval was measured using AcQKNowledge (Biopac Acquisition System). Data were analysed from the three consecutive phases of the parabolic flight by manual marking each phase upon the pilot indication. The beginning and end of each phase were defined as follows: $>1.6 \mathrm{~g}$ for hypergravity and $<0.1 \mathrm{~g}$ for microgravity. Time domain HRV indices including mean RR interval (mean RR), standard deviation of the intervals (RRSD, a measure of the total autonomic nervous system including sympathetic and parasympathetic), and square root of the mean squared difference of successive intervals (rMSSD, a report of parasympathetic modulation) were determined $^{12}$.

\section{QTc and short term QT variability (STVQT)}

Thirty consecutive beat-to-beat QT intervals were manually analysed from the recorded data using ACQKNOWLEDGE. QT intervals were measured from the onset of QRS complex to the end of $\mathrm{T}$ wave and then were corrected to QTc. QT variability was evaluated from Poincaré plot of each QT value against its former value. STVQT is determined from mean orthogonal distance of the diagonal to the point of Poincaré plot ${ }^{13}$ as follows:

$$
\text { STVQT }=\sum \frac{\left|D_{n+1}-D_{n}\right|}{30 \sqrt{2}},
$$

where $D$ represents the duration of QT.

Data are presented as mean \pm SEM. Repeated measures of two-way ANOVA were used for determining overall significant difference among the various phases of gravitational change, and Kolmogorov test was then used to determine the difference between phases. The 0.05 level of probability was used to indicate a significant level of analysis.

\section{RESULTS}

Guinea pigs treated with caffeine and doxorubicin demonstrated significant decrease in body weight and percent body weight gain $(614 \pm 14 \mathrm{~g}$ versus $654 \pm 12 \mathrm{~g}$ and $20.4 \pm 0.6 \mathrm{~g}$ versus $26.8 \pm 1.9 \mathrm{~g}$, respectively) compared to control. Histological examination clearly demonstrated cytoplasmic vacuolation, myofibrillar degeneration, and inflammatory cell infiltration in heart of CM guinea pigs (Fig. 1). However, one set of data from CM guinea pigs were discarded because the animal had no confirmation of ECG change from control at normal gravity (Fig. 3a). Thus data from only three CM guinea pigs were used to compare in the study.

As shown in Fig. 2, comparison between the control and CM groups at normal gravity as well as during gravitational transition showed no differences in heart rate (164 \pm 7 versus $159 \pm 5 \mathrm{bpm})$ and mean RR interval (368 \pm 16 versus $379 \pm 11 \mathrm{~ms}$ ). Significant decreases in RRSD and rMSSD values in CM group ( $1.34 \pm 0.11 \mathrm{~ms} ; 2.38 \pm 0.23 \mathrm{~ms})$ as compared to controls $(2.01 \pm 0.14 \mathrm{~ms} ; 3.13 \pm 0.23 \mathrm{~ms})$ (Fig. 3a,b) indicates a lower parasympathetic/sympathetic ratio. In addition, QTc of CM guinea pigs ( $374 \pm 6 \mathrm{~ms}$ ) is significantly prolonged compared to 

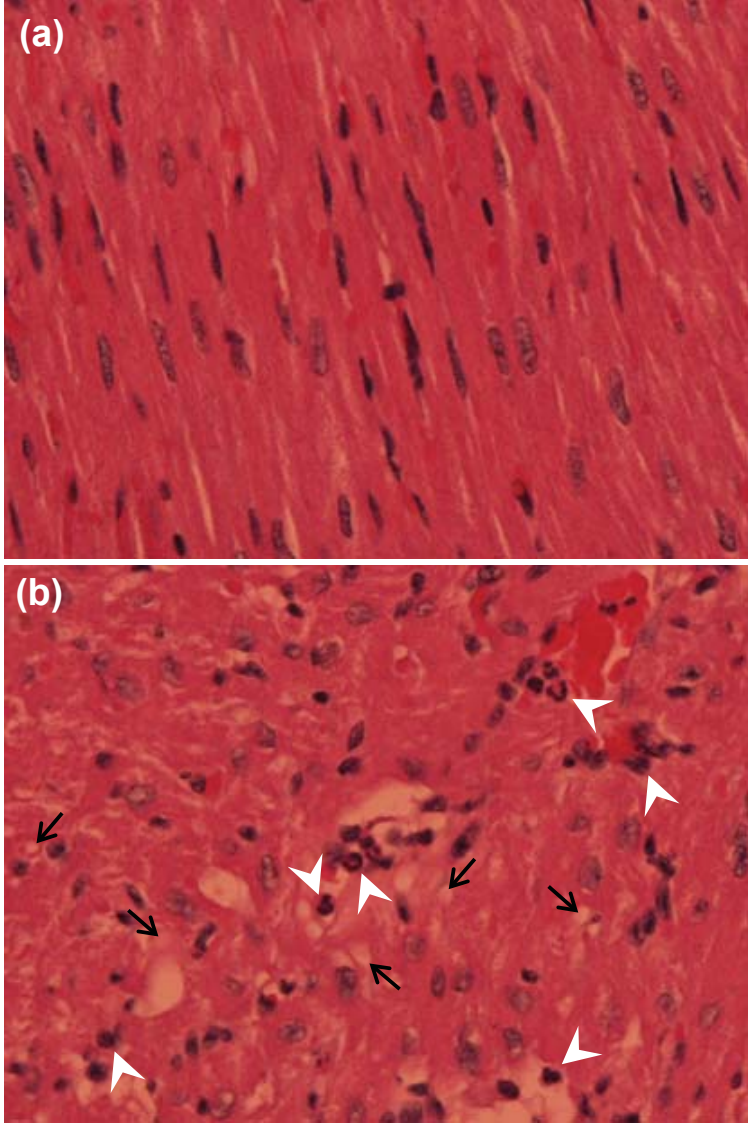

Fig. 1 Representative histological images of ventricular myocardium of (a) healthy and (b) doxorubicin-induced cardiomyopathic guinea pigs. Black arrows show cytoplasmic vacuolation while arrow heads indicate polymorphonuclear cells.

that of control (353 $\pm 5 \mathrm{~ms}$ ) (Fig. 3d), suggesting an increased risk of torsades de pointes arrhythmias following induction of $\mathrm{CM}^{14}$.

Data collected during transition to $1.8 \mathrm{~g}$ showed a decrease in rMSSD in controls $(2.10 \pm 0.10 \mathrm{~ms})$ and be reversed at $0 g$ (Fig. $3 \mathrm{~b}$ ) indicating a reduction of parasympathetic tone by hypergravity. However, this change was not observed in CM group suggesting a probable persistent low level of parasympathetic activity. On the other hand, there was a significant suppression of RRSD, implying an enhancement of sympathetic activity, in CM guinea pigs at hypergravity $(0.96 \pm 0.11 \mathrm{~ms})$, which was reversed under microgravity $(1.33 \pm 0.05 \mathrm{~ms})$ (Fig. 3a). CM-induced decrease in RRSD detected at normal gravity was still present throughout the hyper- and microgravity stages. Thus enhanced sympathetic activity of CM group was present at (a)

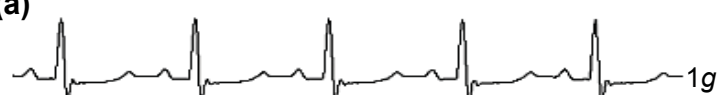

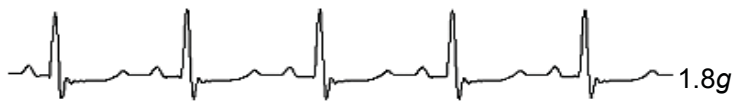

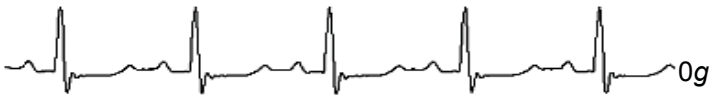

(b)

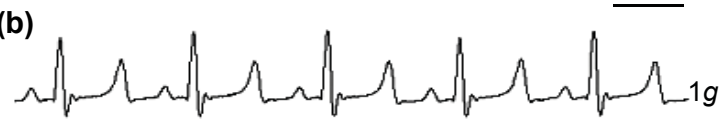

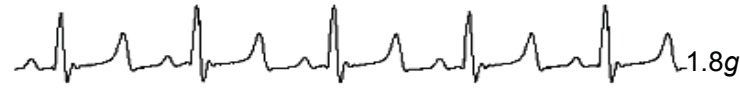

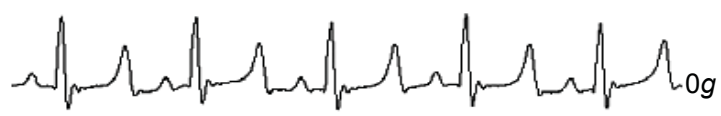

$\underline{200 \mathrm{~ms}}$

Fig. 2 Electrocardiograms in representative (a) healthy and (b) doxorubicin-induced cardiomyopathic guinea pigs during one parabola.

both normal and hypergravity. In contrast to RRSD, a significant suppression of the QTc was observed in CM guinea pigs by microgravity (Fig. 3c), which could cancel out CM-increased risk of torsades de pointes arrhythmias.

From Poincaré plot of QT values, the derived STVQT data of control group during microgravity $(0.13 \pm 0.01 \mathrm{~ms})$ are significantly increased compared to those measured under normal gravity $(0.11 \pm 0.00 \mathrm{~ms})$ and hypergravity $(0.10 \pm 0.01 \mathrm{~ms})$ phases (Fig. 3d). This increased STVQT value suggests a possibility of reduction in repolarization reserve under microgravity ${ }^{15}$. In contrast to control group, CM guinea pigs demonstrated no effect of microgravity on STVQT.

In addition, ECG tracer of CM guinea pigs at normal gravity showed a significant increase in QRS interval compared to controls $(66.2 \pm 2.4$ versus $54.2 \pm 3.1 \mathrm{~ms}$ ). However, gravitational transition had no influence on the QRS interval of either group. There was also no influence of either cardiomyopathy or gravitational transition on the $T_{\text {peak }}-T_{\text {end }}$ interval. All ECG parameters in both control and $\mathrm{CM}$ groups remained unchanged throughout fifteen repetitive cycles of parabolic flight (data not shown). 

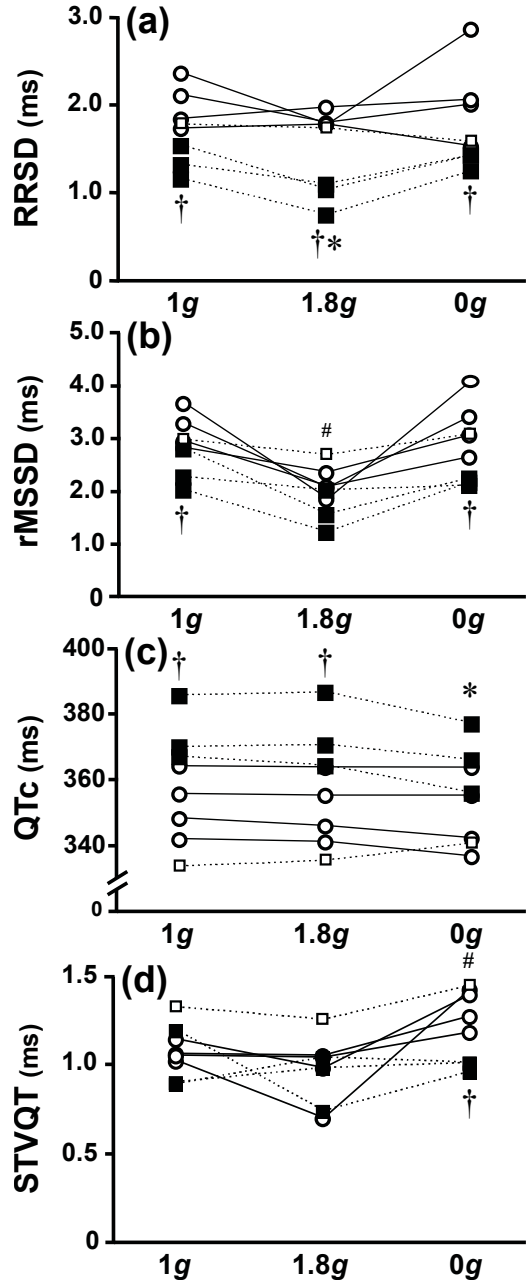

Fig. 3 Effect of gravity on heart rate variability and QT interval of healthy (o) and cardiomyopathic ( $\mathbf{a})$ guinea pigs. (a) RRSD, (b) rMSSD, (c) QTc, (d) STVQT. Values are expressed for individual animals. ${ }^{\dagger}$ significant difference compared with control group, $p<0.05$. \#, * significant difference compared to normal gravity of the control and cardiomyopathic groups, respectively, $p<0.05$. $\square$ represents a doxorubicin-injected guinea pig but no sign of ECG abnormality.

\section{DISCUSSION}

The present study evaluates the impact of changes in gravity created during parabolic flight on heart rate variability of $\mathrm{CM}$ guinea pigs. Doxorubicin and caffeine treatment caused an enhancement of sympathetic activity in CM guinea pigs, which could lead to an increased risk of arrhythmia, especially in hypergravity condition. In addition, the increase in STVQT during microgravity observed only in control but not in CM group implies that elevated sympathetic activity is restricted to damaged heart. Interestingly, the reduction in QTc of CM heart under microgravity suggests that microgravity might help reduce risk of tachyarrhythmia.

During space flight, an astronaut experiences short-term hypergravity during liftoff and landing. As with healthy individual in supine position, increased $g$ force exerts no effect on autonomic activity to the heart in anaesthetized healthy guinea pigs $^{16-18}$. Interestingly, with less haemodynamic changes in CM group who has impaired parasympathetic but increased sympathetic activity, a further reduction in parasympathetic/sympathetic ratio during hypergravity would then potentially enhance load of the heart. This data also points to another unclear mechanism activating autonomic response during gravitational transition besides shift of vascular fluid. Changes in cerebral blood flow velocity and cranial fluid volume during high $g{ }^{19}$ might be one possible mechanism affecting the cardiovascular centre.

Surprisingly, an increase in STVQT of control guinea pigs at microgravity indicates decreased repolarization ability, which suggests a potential increase in cardiac arrhythmia ${ }^{20}$. An increased variability in beat-to beat QT interval has recently been reported in humans subjects undergoing 90 days of head-down bed rest, which is widely accepted as being a ground-based analogue of space flight jour$n^{n e y}{ }^{21}$. This supports the possibility of microgravityinduced increased risk of cardiac arrhythmia in a healthy astronaut. However, unaltered STVQT during the parabolic flights in CM guinea pigs could be the effect of hypersympathetic activation in this animal as a reduction of RRSD and previously reported $^{22}$.

Significant reduction of QTc interval in CM animals at microgravity suggests a potential of lowering cardiac efforts in patients with heart disease by being in space. Previous study has demonstrated that doxorubicin induces prolonged QT interval with a reduced repolarization reserve leading to the development of life-threatening proarrhythmia ${ }^{23}$. Hence the inhibition of the slow potassium current $I_{\mathrm{Ks}}$ by doxorubicin ${ }^{24}$ in combination with an increase in $I_{\mathrm{Ca}}$ by hypersympathetic activation may be a potential mechanism in explaining the reduced repolarization reserve in CM group. It is, however, not clear at this point how microgravity induced QTc normalization in CM group despite a still higher sympathetic activity than controls. Whether the QTc normalization is induced by a direct impact of microgravity or not is not clear in this study but an 
evidence showing direct effect of microgravity on $\mathrm{Ca}^{2+} / \mathrm{K}^{+}$current system in the hair cell of semicircular canals ${ }^{25}$ supports the possibility. Further investigation on the ionic events during gravitational transition is in need before a clear conclusion can be drawn.

\section{Limitations}

The amount of data was limited owing to the restricted working space and an inability to manipulate the animal during parabolic flight. All detected changes in the study were immediate responses to gravitational transition in a short period, which might be different from the situation of prolonged exposure to micro- or hypergravity. Moreover, our study was performed using guinea pigs in which gravity may have less effect on blood pool in recumbent position. Data obtained from this study might not completely mimic the response in human especially on standing position.

In conclusion, we found over-activation of sympathetic and parasympathetic signals in cardiomyopathy guinea pig heart compared to control during transition from normal to hyper- to microgravity, which could increase the risk of arrhythmia leading to cardiac sudden death. The reduced risk of arrhythmia observed in CM heart during microgravity thus urges an initial idea of further investigation on the contributed cellular signals of the protective action. The underlying protective mechanisms may provide beneficial basis for future therapeutic approach to heart disease management.

Acknowledgements: We thank Japan Aerospace Exploration Agency (JAXA) for organizing the parabolic flight, Dr Sawat Tantiphanwadi, NSTDA, Thailand for coordinating the project with JAXA, Dr Noppadol Larbcharoensub and Dr Pornsiri Pitchakarnand for histopathological technical support and evaluation, Dr Monthip Tiensuwan for statistical guidance, and Dr Prapon Wilairat for critical reading of the manuscript. This study was supported by NSTDA, and the Faculty of Medicine Ramathibodi Hospital and the Faculty of Science, Mahidol University.

\section{REFERENCES}

1. Leach CS, Inners LD, Charles JB (1991) Changes in total body water during spaceflight. $J$ Clin Pharmacol 31, 1001-6.

2. Diedrich A, Drescher J, Nalishitj V, Kirchner F (1994) Acute effects of simulated microgravity on heart rate variability. J Gravit Physiol 1, 35-6.

3. Caiani EG, Sugeng L, Weinert L, Husson S, Bailliart O, Capderou A, Lang RM, Vaida P (2004) Feasibility of real-time 3D echocardiography in weightlessness during parabolic flight. $J$ Gravit Physiol 11, 235-6.

4. Capderou A, Bailliart O, Maison-Blanche P, Kedra AW, Atkov O, Techoueyres P, Lachaud JL, Vaïda P (2001) Parasympathetic activity during parabolic flight, effect of LBNP during microgravity. Aviat Space Environ Med 72, 361-7.

5. Petersen LG, Damgaard M, Petersen JC, Norsk P (2011) Mechanisms of increase in cardiac output during acute weightlessness in humans. J Appl Physiol 111, 407-11.

6. Somody L, Fagette S, Frutoso J, Gharib C, Gauquelin $\mathrm{G}$ (1998) Recording heart rate and blood pressure in rats during parabolic flight. Life Sci 63, 851-7.

7. Ponikowski P, Anker SD, Chua TP, Szelemej R, Piepoli M, Adamopoulos S, Webb-Peploe K, Harrington D, et al (1997) Depressed heart rate variability as an independent predictor of death in chronic congestive heart failure secondary to ischemic or idiopathic dilated cardiomyopathy. Am J Cardiol 79, 1645-50.

8. Sanderson JE, Yeung LYC, Yeung DTK, Kay RLC, Tomlinson B, Critchley JAJH, Woo KS, Bernardi L (1996) Impact of changes in respiratory frequency and posture on power spectral analysis of heart rate and systolic blood pressure variability in normal subjects and patients with heart failure. Clin Sci 91, 35-43.

9. Gabrielsen A, Sørensen VB, Pump B, Galatius S, Videbæk R, Bie P, Warberg J, Christensen NJ, et al (2000) Cardiovascular and neuroendocrine responses to water immersion in compensated heart failure. Am J Physiol Heart Circ Physiol 279, H1931-40.

10. Desai N, Raghunandan DS, Mallavarapu M, Berger RD, Yeragani VK (2004) Beat-to-beat heart rate and QT variability in patients with congestive cardiac failure: blunted response to orthostatic challenge. Ann Noninvasive Electrocardiol 9, 323-9.

11. Hosenpud JD, Wright J, Simpson L, Abramson JJ (1995) Caffeine enhances doxorubicin cardiac toxicity in an animal model. $J$ Card Fail 1, 155-60.

12. Sztajzel J (2004) Heart rate variability: a noninvasive electrocardiographic method to measure the autonomic nervous system. Swiss Med Wkly 134, 514-22.

13. Thomsen MB, Verduyn SC, Stengl M, Beekman JD, de Pater G, van Opstal J, Volders PG, Vos MA (2004) Increased short-term variability of repolarization predicts $d$-sotalol-induced torsades de pointes in dogs. Circulation 110, 2453-9.

14. Sides GD (2002) QT interval prolongation as a biomarker for torsades de pointes and sudden death in drug development. Dis Markers 18, 57-62.

15. Varró A, Baczkó I (2011) Cardiac ventricular repolarization reserve: a principle for understanding drug-related proarrhythmic risk. Br J Pharmacol 164, 14-36.

16. Beckers F, Seps B, Ramaekers D, Verheyden B, Aubert 
AE (2003) Parasympathetic heart rate modulation during parabolic flights. Eur J Appl Physiol 90, 83-91.

17. Liu J, Verheyden B, Beckers F, Aubert AE (2012) Haemodynamic adaptation during sudden gravity transitions. Eur J Appl Physiol 112, 79-89.

18. Seps B, Beckers F, Aubert AE (2002) Heart rate variability during gravity transitions. In: Computers in Cardiology, 2002, vol. 29, IEEE, pp 433-6.

19. Kawai Y, Puma SC, Hargens AR, Murthy G, Warkander D, Lundgren CEG (1997) Cerebral blood flow velocity and cranial fluid volume decrease during $+\mathrm{G}_{\mathrm{Z}}$ acceleration. $J$ Gravit Physiol 4, 31-4.

20. Pajouh M, Wilson LD, Poelzing S, Johnson NJ, Rosenbaum DS (2005) $I_{\mathrm{Ks}}$ blockade reduces dispersion of repolarization in heart failure. Heart Rhythm 2, 731-8.

21. Sakowski C, Starc V, Smith SM, Schlegel TT (2011) Sedentary long-duration head-down bed rest and ECG repolarization heterogeneity. Aviat Space Environ Med 82, 416-23.

22. Moguilevski V, Oliver J, McGrath BP (1995) Sympathetic regulation in rabbits with heart failure: experience using power spectral analysis of heart rate variability. Clin Exp Pharmacol Physiol 22, 475-7.

23. Milberg P, Fleischer D, Stypmann J, Osada N, Mönnig G, Engelen MA, Bruch C, Breithardt G, et al (2007) Reduced repolarization reserve due to anthracycline therapy facilitates torsade de pointes induced by $I_{\mathrm{Kr}}$ blockers. Basic Res Cardiol 102, 42-51.

24. Ducroq J, Moha ou Maati H, Guilbot S, Dilly S, Laemmel E, Pons-Himbert C, Faivre JF, Bois P, et al (2010) Dexrazoxane protects the heart from acute doxorubicin-induced QT prolongation: a key role for $I_{\mathrm{Ks}}$. Br J Pharmacol 159, 93-101.

25. Martini M, Canella R, Leparulo A, Prigioni I, Fesce $\mathrm{R}$, Rossi ML (2009) Ionic currents in hair cells dissociated from frog semicircular canals after preconditioning under microgravity conditions. Am J Physiol Regul Integr Comp Physiol 296, R1585-97. 